\title{
SINGULAR POLYNOMIALS FOR FINITE REFLECTION GROUPS
}

\author{
C. F. DUNKL, M. F. E. DE JEU, AND E. M. OPDAM
}

\begin{abstract}
The Dunkl operators involve a multiplicity function as parameter. For generic values of this function the simultaneous kernel of these operators, acting on polynomials, is equal to the constants. For special values, however, this kernel is larger. We determine these singular values completely and give partial results on the representations of $G$ that occur in this kernel.
\end{abstract}

\section{INTRODUCTION AND NOTATIONS}

Let $\mathfrak{a}$ be a real vector space of finite dimension $N$, equipped with an inner product $(\cdot, \cdot)$. Let $G \subset O(\mathfrak{a}(\cdot, \cdot))$ be a finite (real) reflection group. We may and will assume that $(\cdot, \cdot)$ is $G$-invariant. Let $R$ be the corresponding root system, where we will assume that $(\alpha, \alpha)=2$ for all $\alpha \in R$. Choose and fix a positive system $R_{+}$in $R$.

Let $\mathscr{P}=\mathbf{C}[\mathfrak{a}]$ denote the polynomial functions on $\mathfrak{a} . \mathscr{P}$ has a natural grading $\mathscr{P}=\bigoplus_{n \geq 0} \mathscr{P}_{n}$; we let $\mathscr{P}_{+}=\bigoplus_{n \geq 1} \mathscr{P}_{n}$. There is a natural representation of $G$ in $\operatorname{Aut}_{\mathbf{C}}(\mathscr{P})$, given by

$$
(g \cdot f)(x):=f\left(g^{-1} x\right) \quad(g \in G, f \in \mathscr{P}, x \in \mathfrak{a}) .
$$

The $G$-action preserves the degree.

Any $\xi \in \mathfrak{a}$ defines an element $\xi^{*} \in \operatorname{Hom}_{\mathbf{C}}(\mathfrak{a}, \mathbf{C})=\mathfrak{a}^{*}=\mathscr{P}_{1}$ by $\xi^{*}(\eta)=$ $(\xi, \eta) \quad(\eta \in \mathfrak{a})$. Let $K$ be the complex vectorspace of $\mathbf{C}$-valued $G$-invariant functions on $R$. An element of $K$ is called a multiplicity function (the reason for this terminology is a connection of the theory of Dunkl operators with the harmonic analysis for the Cartan motion group; the values $k_{\alpha}$ of $k \in K$ are then determined by the multiplicities of the restricted roots). To each $\xi \in \mathfrak{a}$ and $k \in K$ we assign an operator $T_{\xi}(k) \in \operatorname{End}_{\mathbf{C}}(\mathscr{P})$ as follows:

$$
T_{\xi}(k) f=\partial_{\xi} f+\sum_{\alpha \in R_{+}} k_{\alpha}(\alpha, \xi) \frac{f-r_{\alpha} f}{\alpha^{*}} \quad(f \in \mathscr{P}),
$$

where $\partial_{\xi}$ is the directional derivative corresponding to $\xi$ and $r_{\alpha}$ is the reflection in the hyperplane orthogonal to $\alpha$.

These operators were introduced in [D2]. In that paper it was shown that $\left\{T_{\xi}(k) \mid \xi \in \mathfrak{a}\right\}$ is a commuting family of operators, for all fixed $k \in K$. They are homogeneous of degree -1 and $G$-equivariant:

$$
g \circ T_{\xi}(k) \circ g^{-1}=T_{g \xi}(k) \quad(\xi \in \mathfrak{a}, g \in G) .
$$

Received by the editors February 4, 1994.

1991 Mathematics Subject Classification. Primary 20F55, 33C80, 33C35, 20 C30.

During the preparation of this paper Dunkl was partially supported by NSF grant DMS-9103214. 
For $k=0$, Dunkl's operators reduce to the ordinary directional derivatives, so the simultaneous kernel of the $T_{\xi}(k) \quad(\xi \in \mathfrak{a})$ consists of the constants alone. For generic $k \in K$ this is still true, but there exist exceptional $k$ for which this simultaneous kernel is strictly larger than the constants. This paper is concerned with the study of such singular values. Let us introduce some terminology. For $k \in K$, put $S(k)=\left\{p \in \mathscr{P}_{+} \mid T_{\xi}(k) p=0 \quad \forall \xi \in \mathfrak{a}\right\}$. If $S(k) \neq\{0\}$, then $k$ is called singular, and regular otherwise. We use the notation $K^{\text {sing }}$ (resp. $K^{\text {reg }}$ ) for the set of all singular (resp. regular) $k \in K$. Similarly, a polynomial $p \in \mathscr{P}_{+}$ is called singular if $p \in S(k)$ for some $k \in K$. Put $S=\sum_{k \in K} S(k)$. As a consequence of the homogeneity and the $G$-equivariance of the $T_{\xi}(k)$ one may split $S$ according to homogeneous degree and $G$-type: $S=\bigoplus_{n \geq 1, \tau \in \widehat{G}} S_{n, \tau}$, where $\widehat{G}$ denotes the dual of $G$. There is an easy first observation on $K^{\text {sing }}$. Let $\left\{e_{1}, \ldots, e_{N}\right\}$ be an orthonormal basis for $\mathfrak{a}$. Then

$$
\sum_{i=1}^{N} e_{i}^{*} T_{e_{i}}(k)=\sum_{i=1}^{N} e_{i}^{*} \partial_{e_{i}}+\sum_{\alpha \in R_{+}} k_{\alpha}\left(1-r_{\alpha}\right) .
$$

Due to the $G$-invariance of $k, \sum_{\alpha \in R_{+}} k_{\alpha}\left(1-r_{\alpha}\right)$ is a central element in the group algebra of $G$. Hence it acts as a scalar $\lambda_{\tau}(k)$ in any irreducible representation $\tau \in \widehat{G}$. If $k_{\alpha}=k \in \mathbf{C} \quad(\forall \alpha \in R)$, then it is easy to see that $\lambda_{\tau}\left(k \cdot 1_{R}\right)=$ $k \sum_{\alpha \in R_{+}}\left(1-\chi_{\tau}\left(r_{\alpha}\right) / \chi_{\tau}(1)\right)$, where $\chi_{\tau}$ denotes the character of $\tau$. Now if $p \in$ $S(k)$ is of isotype $\tau$ and homogeneous degree $m$, then $p$ is annihilated by the operator in (1.2); this gives the inclusion $S_{m, \tau} \subset S\left(-m / \lambda_{\tau}\left(1_{R}\right)\right)$ in the case of equal $k_{\alpha}$. In other words, we can determine $K^{\text {sing }}$ completely if we know the embedding degrees of all $\tau \in \widehat{G}$ in $S$. One might hope that $K^{\text {sing }}=$ $\left\{-m / \lambda_{\tau}\left(1_{R}\right) \mid m>0, \tau \in \widehat{G}, \tau\right.$ occurs in $\left.\mathscr{P}_{m}\right\}$, but this is false (the easiest counterexample is furnished by $\left.G=S_{4}\right)$. Let us note that $\lambda_{\tau}\left(1_{R}\right)$ is an integer; this follows from general representation theory of finite groups. It is easy to see that this integer is nonpositive and equal to zero iff $\tau=$ triv. So $K^{\text {sing }}$ will consist of strictly negative rationals in the case of equal $k_{\alpha}$.

One of the reasons for our interest in $S$ is the fact that $S(k)$ turns out to be the only obstruction for the existence of a linear isomorphism $V(k): \mathscr{P} \mapsto \mathscr{P}$, homogeneous of degree 0 , that intertwines the action of $T_{\xi}(k)$ and the ordinary derivative $\partial_{\xi}$ on $\mathscr{P}$ (see Corollary 3.5). There are many other different descriptions for $S$ or $K^{\text {sing }}$ and their role with regard to the Dunkl operators. For example, $K^{\text {sing }}$ is also the set of poles of the simultaneous $T_{\xi}(k)$-eigenfunction $\operatorname{Exp}_{G}(\lambda, k, x)$, considered as a function of $k \in K$ (see [O]). Using monodromy arguments this set of poles was described in terms of the zeros of the Poincare polynomial of the coinvariant algebra of $G$ [O, Theorem 9.7]. This leads to an explicit description of $K^{\text {sing }}$. If $k_{\alpha}=k \quad \forall \alpha \in R$, then the result is

$$
K^{\text {sing }}=\bigcup_{m \geq 1} \bigcup_{i=1}^{N} \bigcup_{j=1}^{d_{i}-1}\left\{\frac{j}{d_{i}}-m\right\},
$$

where the $d_{i}$ are the primitive degrees of $G$. Yet another meaning for this set $K^{\text {sing }}$ is the following. As a consequence of the commutativity of the $T_{\xi}(k)$ for fixed $k$, there exists an algebra homomorphism from $\mathscr{P}$ to $\operatorname{End}_{\mathbf{C}}(\mathscr{P})$ which sends $\xi^{*}$ to $T_{\xi}(k)$ and 1 to the identity. If we define the bilinear form $(\cdot, \cdot)_{k}$ 
on $\mathscr{P} \times \mathscr{P}$ by $(p, q)_{k}=(p(T(k))(q))(0)$, then $K^{\text {sing }}$ is the set of all values for which this form is degenerate.

The purpose of this paper is twofold. First of all, we explain these different interpretations of the space $S$ and the set $K^{\text {sing }}$. Secondly, we try to get a better understanding of the structure of $K^{\text {sing }}$ with regard to the representation theory of $G$. The optimal result in this direction would be a list of irreducibles $\tau \in \widehat{G}$ that can occur in $S$, and for each of these a description of the function $m \mapsto \operatorname{dim}_{\mathbf{C}}\left(S_{m, \tau}\right)$. As yet this seems out of reach, but we will be able to give estimates for $\operatorname{dim}_{C}\left(S_{m, \tau}\right)$ entirely in terms of decomposition numbers for Hecke algebras.

\section{THE EXISTENCE OF INTERTWINING OPERATORS}

The present section is concerned with the existence of operators that intertwine the action of two sets of operators in a graded vectorspace. The presence of a grading for which the various actions are homogeneous enables one to use induction. This fact and an additional assumption (formulated in the language of differential forms) are surprisingly powerful tools for the construction of certain intertwining operators. The underlying ideas were used more or less parenthetically in [D3]; this section can be regarded as an abstraction and extension of the proof of Theorem 3.11 in [loc. cit.]. The results show that, in the context of this section, there is an equivalence between the commutativity of sets of operators, the triviality of the first cohomology-group of a certain complex and the existence of intertwining operators. We will use this equivalence to give a new proof of the commutativity of Dunkl's operators and the existence of the intertwining operator (see [loc. cit.]).

Let $L$ be a vectorspace with a grading $L=\bigoplus_{i=0}^{\infty} L_{i}$. Put $L_{-1}=0$. Let $k$ be a positive integer and suppose $\left\{\partial_{j}\right\}_{j=1}^{k}$ are linear operators in $L$ such that

(i) $\partial_{j} L_{i} \subset L_{i-1}(j=1, \ldots, k ; i=0,1 \ldots)$.

(ii) $\bigcap_{j=1}^{k} \operatorname{Ker}\left(\partial_{j}\right)=L_{0}$.

A sequence $\left\{l_{i}\right\}_{i=1}^{k}$ in $L$ will be called a $\partial$-closed 1 -form if $\partial_{j} l_{i}=\partial_{i} l_{j}$ $(i, j=1, \ldots, k)$; it is a $\partial$-exact 1 -form if there exists $l \in L$ such that $\partial_{i} l=l_{i}$ $(i=1, \ldots, k)$. Suppose in addition that

(iii) every $\partial$-closed 1 -form is $\partial$-exact.

Theorem 2.1. Let $\left\{\delta_{j}\right\}_{j=1}^{k}$ be linear operators in $L$ such that

(i') $\delta_{j}\left(L_{i}\right) \subset L_{i-1}(j=1, \ldots, k ; i=0,1 \ldots)$;

(ii') the $\delta_{j}$ commute.

Let $\left\{\partial_{j}\right\}_{j=1}^{k}$ be linear operators in $L$ satisfying (i), (ii), and (iii) above and let $A: L_{0} \mapsto L_{0}$ be linear. Then there exists a unique linear map $B: L \mapsto L$ such that:

(a) $B$ extends $A$;

(b) $\partial_{j} B=B \delta_{j} \quad(j=1, \ldots, k)$;

(c) $B L_{i} \subset L_{i}(i=0,1, \ldots)$.

If $A$ is injective and $\bigcap_{j=1}^{k} \operatorname{Ker}\left(\delta_{j}\right)=L_{0}$, then $B$ is injective.

Proof. Existence. We will construct a family of operators $B_{i}: L_{i} \mapsto L_{i} \quad(i=$ $0,1, \ldots)$ such that:

( $\alpha) B_{0}=A$;

(ß) $\partial_{j} B_{i}=B_{i-1} \delta_{j} \quad(j=1, \ldots, k ; i=1,2, \ldots)$; 
(y) $B_{i} L_{i} \subset L_{i}(i=0,1, \ldots)$.

Assuming for the moment that this has been done, we can take $\left.B\right|_{L_{i}}=B_{i}$ and extend by linearity to obtain an endomorphism of $L$, which satisfies (a), (b), and (c).

The construction is by induction. Put $B_{0}=A$. Next, let $l \in L_{1}$. Consider the 1 -form $\left\{A \delta_{i} l\right\}_{i=1}^{k}$. Since $A \delta_{i} l \in L_{0}$, this 1 -form is trivially $\partial$-closed by (i), so there exists $l^{\prime} \in L$ such that $\partial_{i} l^{\prime}=A \delta_{i} l \quad(i=1, \ldots, k)$. Properties (i) and (ii) imply that any such $l^{\prime}$ is in $L_{0} \oplus L_{1}$. By (i), we may take $l^{\prime}$ in $L_{1}$ and it is then uniquely determined as a consequence of (ii). We can thus define $B_{1} l$ to be the unique element in $L_{1}$ satisfying $\partial_{i} B_{1} l=A \delta_{i} l \quad(i=1, \ldots, k)$. The uniqueness makes it clear that $B_{1}: L_{1} \mapsto L_{1}$ is linear. For the induction step, assume that $B_{0}, \ldots, B_{d}(d \geq 1)$ have been constructed and satisfy $(\alpha),(\beta)$, and $(\gamma)$ (for $i=0, \ldots, d)$. Let $l \in L_{d+1}$ and consider the 1 -form $\left\{B_{d} \delta_{i} l\right\}_{i=1}^{k}$. Since the $\delta_{i}$ commute, we have, using the induction hypothesis

$$
\partial_{j} B_{d} \delta_{i} l=B_{d-1} \delta_{j} \delta_{i} l=B_{d-1} \delta_{i} \delta_{j} l=\partial_{i} B_{d} \delta_{j} l \text {. }
$$

Hence the form is $\partial$-closed and there exists $l^{\prime} \in L$ such that $\partial_{i} l^{\prime}=B_{d} \delta_{i} l$ $(i=1, \ldots, k)$. The same argument as above shows that we can determine $l^{\prime}$ uniquely by requiring it to be in $L_{d+1}$. Again, the map $B_{d+1}: L_{d+1} \mapsto L_{d+1}$, defined by $B_{d+1} l=l^{\prime}$ is linear and by construction we have $\partial_{i} B_{d+1} l=\partial_{i} l^{\prime}=$ $B_{d} \delta_{i} l \quad(i=1, \ldots, k)$. This completes the induction step.

Uniqueness. Let $B^{\prime}$ be any other operator in $L$ satisfying (a), (b), and (c) in the Theorem. Put $B_{d}^{\prime}=\left.B^{\prime}\right|_{L_{d}}$. We prove by induction that $B_{d}^{\prime}=B_{d}$ $(d=0,1, \ldots)$. For $d=0$, this is just (a). Assuming $B_{i}^{\prime}=B_{i}(i=0, \ldots, d)$, we have for $l \in L_{d+1}$ arbitrary

$$
\partial_{j}\left(B_{d+1}-B_{d+1}^{\prime}\right) l=\left(B_{d}-B_{d}^{\prime}\right) \delta_{j} l=0 \quad(j=1, \ldots, k),
$$

so $\left(B_{d+1}-B_{d+1}^{\prime}\right) l \in L_{0}$ by (ii). But then $\left(B_{d+1}-B_{d+1}^{\prime}\right) l \in L_{0} \cap L_{d+1}$ by (c), which is the zero subspace since $d+1 \geq 1$.

We now prove the last statement of the theorem. Put $L^{d}=\bigoplus_{i=0}^{d} L_{i} \quad(d=$ $0,1, \ldots)$. We prove, again by induction, that $B$ is injective on $L^{d}$. For $d=0$, this is the injectivity of $A$. Assuming the statement for $i=0, \ldots, d$, let $l \in L^{d+1}$ and suppose $B l=0$. Then, by (b), $B \delta_{j} l=0 \quad(j=1, \ldots, k)$. Since $\delta_{j} l \in L^{d}, \delta_{j} l=0 \quad(j=1, \ldots, k)$ by the induction hypothesis. So, by the assumption on the simultaneous kernel of the $\delta_{j}, l$ is in $L_{0}$. But then $A l=B l=0$, so $l=0$.

Corollary 2.2. Let $L$ and the $L_{i}$ be as above and suppose in addition that $\operatorname{dim}\left(L_{i}\right)<\infty \quad(i=1,2, \ldots)$. Suppose that $\left\{\partial_{j}\right\}_{j=1}^{k}$ are linear operators such that:

(i) $\partial_{j} L_{i} \subset L_{i-1}(j=1, \ldots, k ; i=0,1, \ldots)$;

(ii) $\bigcap_{j=1}^{k} \operatorname{Ker}\left(\partial_{j}\right)=L_{0}$;

(iii) every $\partial$-closed 1-form is $\partial$-exact;

(iv) the $\partial_{j}$ commute.

Suppose $\left\{\delta_{j}\right\}_{j=1}^{k}$ are linear operators in $L$ such that

(i') $\delta_{j} L_{i} \subset L_{i-1}(j=1, \ldots, k ; i=0,1, \ldots)$;

(ii') $\bigcap_{j=1}^{k} \operatorname{Ker}\left(\delta_{j}\right)=L_{0}$.

Then the following are equivalent: 
(a) The $\delta_{j}$ commute.

(b) Every $\delta$-closed 1-form is $\delta$-exact.

(c) There exists an automorphism $A$ of $L_{0}$ that extends to an automorphism $B$ of $L$ such that $\partial_{j} B=B \delta_{j} \quad(j=1, \ldots, k)$.

(d) Every automorphism $A$ of $L_{0}$ uniquely extends to an automorphism $B$ of $L$ such that $\partial_{j} B=B \delta_{j} \quad(j=1, \ldots, k)$ and $B L_{i}=L_{i} \quad(i=0,1, \ldots)$.

Proof. $(\mathrm{a}) \Rightarrow(\mathrm{d})$ : Theorem 2.1 applies. Since $B$ is injective on the $L_{i}$, it is an automorphism by the finite dimensions of the $L_{i}$. The implications $(\mathrm{d}) \Rightarrow$ (c) and $(\mathrm{c}) \Rightarrow(\mathrm{a})$ are obvious.

(b) $\Rightarrow(\mathrm{d})$ : Apply Theorem 2.1 with the roles of the $\partial_{j}$ and $\delta_{j}$ interchanged. Again the finite dimensions of the $L_{i}$ ensure that $B$ is an automorphism.

(c) $\Rightarrow(b)$ : This follows from (iii).

\section{THE PERTURBED DE RHAM COMPLEX}

We consider the differential forms on $\mathfrak{a}$ with polynomial coefficients, on which we introduce a tentative boundary operator $d(k)$ (based on the Dunkl operators). A calculation and subsequent appeal to the results of the previous section will enable us to conclude that the Dunkl operators commute; it is then immediate that $d(k)$ is a boundary operator. We conclude with an alternative characterization of singular polynomials.

For $l=0,1, \ldots$, let $K^{l}=\mathscr{P} \otimes_{\mathbf{C}} \Lambda_{l} \mathfrak{a}^{*}$ (this $K^{l}$ has no relation to the space $K$ of multiplicity functions) and put $K^{\bullet}=\bigoplus_{l=0}^{N} K^{l}$. Let $\left\{e_{1}, \ldots, e_{N}\right\}$ be an orthonormal basis for $\mathfrak{a}$. We will denote a typical generator of $K^{l}$ by $p \otimes d e_{i_{1}}^{*} \wedge \cdots \wedge d e_{i_{l}}^{*}$, where $p \in \mathscr{P}$ is a polynomial. An element $g \in G$ acts on $K^{l}$ as follows:

$$
(g \otimes g) \cdot\left(p \otimes d e_{i_{1}}^{*} \wedge \cdots \wedge d e_{i_{l}}^{*}\right)=(g \cdot p) \otimes d\left(g \cdot e_{i_{1}}^{*}\right) \wedge \cdots \wedge d\left(g \cdot e_{i_{l}}^{*}\right) .
$$

We introduce operators $d(k): K^{l} \mapsto K^{l+1}$ by the action on generators:

$$
d(k)\left(p \otimes d e_{i_{1}}^{*} \wedge \cdots \wedge d e_{i_{l}}^{*}\right)=\sum_{j=1}^{N}\left(T_{e_{j}}(k) p\right) \otimes d e_{j}^{*} \wedge d e_{i_{1}}^{*} \wedge \cdots \wedge d e_{i_{l}}^{*} .
$$

Lemma 3.1. $d(k)$ commutes with the G-action.

Proof. The well-known fact that $d(0)$ commutes with the action of $G L(\mathfrak{a})$ follows from an easy computation which is based on the linearity of the map $\xi \mapsto \partial_{\xi}$ and on the $G L(\mathfrak{a})$-equivariance of the ordinary directional derivatives. Hence, the same computation applies for $d(k)$ and $G$, using the $G$ equivariance of the $T_{\xi}(k)$.

We recall the definition of the Koszul-operator $\partial: K^{l} \mapsto K^{l-1}$ :

$\partial\left(p \otimes d e_{i_{1}}^{*} \wedge \cdots \wedge d e_{i_{l}}^{*}\right)=\sum_{r=1}^{l}(-1)^{r+1} e_{i_{r}}^{*} p \otimes d e_{i_{1}}^{*} \wedge \cdots \wedge d e_{i_{r-1}}^{*} \wedge d e_{i_{r+1}}^{*} \wedge \cdots \wedge d e_{i_{l}}^{*}$.

Proposition 3.2. On $K^{l}$, we have

$$
(\partial d(k)+d(k) \partial)=l(1 \otimes 1)+\left(\sum_{j=1}^{N} e_{j}^{*} \partial e_{j}\right) \otimes 1+\sum_{\alpha \in R_{+}} k_{\alpha}\left(1 \otimes 1-r_{\alpha} \otimes r_{\alpha}\right) .
$$


Proof. Let $\omega=p \otimes d e_{i_{1}}^{*} \wedge \cdots \wedge d e_{i_{l}}^{*} \in K^{l}$. We calculate as follows:

$(\partial d(k)+d(k) \partial) \omega$

$$
\begin{aligned}
= & \partial\left(\sum_{j=1}^{N} T_{e_{j}}(k) p \otimes d e_{j}^{*} \wedge d e_{i_{1}}^{*} \wedge \cdots \wedge d e_{i_{l}}^{*}\right) \\
& +d(k)\left(\sum_{r=1}^{l}(-1)^{r+1} e_{i_{r}}^{*} p \otimes d e_{i_{1}}^{*} \wedge \cdots \wedge d e_{i_{r-1}}^{*} \wedge d e_{i_{r+1}}^{*} \wedge \cdots \wedge d e_{i_{l}}^{*}\right) \\
= & \sum_{j=1}^{N} e_{j}^{*} T_{e_{j}}(k) p \otimes d e_{i_{1}}^{*} \wedge \cdots \wedge d e_{i_{l}}^{*} \\
& +\sum_{j=1}^{N} \sum_{r=1}^{l}(-1)^{r} e_{i_{r}}^{*} T_{e_{j}}(k) p \otimes d e_{j}^{*} \wedge d e_{i_{1}}^{*} \wedge \cdots \wedge d e_{i_{r-1}}^{*} \wedge d e_{i_{r+1}}^{*} \wedge \cdots \wedge d e_{i_{l}}^{*} \\
& +\sum_{j=1}^{N} \sum_{r=1}^{l}(-1)^{r+1} T_{e_{j}}(k)\left(e_{i_{r}}^{*} p\right) \otimes d e_{j}^{*} \wedge d e_{i_{1}}^{*} \wedge \cdots \wedge d e_{i_{r-1}}^{*} \wedge d e_{i_{r+1}}^{*} \wedge \cdots \wedge d e_{i_{l}}^{*} .
\end{aligned}
$$

\section{An easy calculation shows that}

$$
\left[T_{e_{j}}(k), e_{i}^{*}\right]=\delta_{i j}+\sum_{\alpha \in R_{+}} k_{\alpha}\left(\alpha, e_{i}\right)\left(\alpha, e_{j}\right) r_{\alpha}
$$

so

$$
\begin{aligned}
(\partial d(k)+d(k) \partial) \omega= & \sum_{j=1}^{N} e_{j}^{*} T_{e_{j}}(k) p \otimes d e_{i_{1}}^{*} \wedge \cdots \wedge d e_{i_{l}}^{*} \\
& +\sum_{j=1}^{N} \sum_{r=1}^{l}(-1)^{r+1}\left(\delta_{j i_{r}}+\sum_{\alpha \in R_{+}} k_{\alpha}\left(\alpha, e_{i_{r}}\right)\left(\alpha, e_{j}\right) r_{\alpha}\right) p \\
= & \sum_{j=1}^{N} e_{j}^{*} T_{e_{j}}(k) p \otimes d e_{j}^{*} \wedge d e_{i_{1}}^{*} \wedge \cdots \wedge d e_{i_{r-1}}^{*} \wedge d e_{i_{i_{1}}}^{*} \wedge \cdots \wedge d e_{i_{l}}^{*} \wedge d e_{i_{l}}^{*} \\
& +\sum_{r=1}^{l}(-1)^{r+1} p \otimes d e_{i_{r}}^{*} \wedge d e_{i_{1}}^{*} \wedge \cdots \wedge d e_{i_{r-1}}^{*} \wedge d e_{i_{r+1}}^{*} \wedge \cdots \wedge d e_{i_{l}}^{*} \\
& +\sum_{\alpha \in R_{+}} k_{\alpha} \sum_{j=1}^{N} \sum_{r=1}^{l}(-1)^{r+1}\left(\alpha, e_{i_{r}}\right)\left(\alpha, e_{j}\right)\left(r_{\alpha} p\right) \\
& \otimes d e_{j}^{*} \wedge d e_{i_{1}}^{*} \wedge \cdots \wedge d e_{i_{r-1}}^{*} \wedge d e_{i_{r+1}}^{*} \wedge \cdots \wedge d e_{i_{l}}^{*} \\
= & \sum_{j=1}^{N} e_{j}^{*} T_{e_{j}}(k) p \otimes d e_{i_{1}}^{*} \wedge \cdots \wedge d e_{i_{l}}^{*}+l \omega \\
& +\sum_{\alpha \in R_{+}} k_{\alpha} \sum_{r=1}^{l}(-1)^{r+1}\left(\alpha, e_{i_{r}}\right)\left(r_{\alpha} p\right) \\
& \otimes d \alpha^{*} \wedge d e_{i_{1}}^{*} \wedge \cdots \wedge d e_{i_{r-1}}^{*} \wedge d e_{i_{r+1}}^{*} \wedge \cdots \wedge d e_{i_{l}}^{*} .
\end{aligned}
$$


Now note that

$$
\begin{aligned}
\left(r_{\alpha} \otimes r_{\alpha}\right)\left(p \otimes d e_{i_{1}}^{*} \wedge \cdots \wedge d e_{i_{l}}^{*}\right) & \\
= & \left(r_{\alpha} p\right) \otimes d e_{i_{1}}^{*} \wedge \cdots \wedge d e_{i_{l}}^{*}+\sum_{r=1}^{l}(-1)^{r}\left(\alpha, e_{i_{r}}\right)\left(r_{\alpha} p\right) \\
& \otimes d \alpha^{*} \wedge d e_{i_{1}}^{*} \wedge \cdots \wedge d e_{i_{r-1}}^{*} \wedge d e_{i_{r+1}}^{*} \wedge \cdots \wedge d e_{i_{l}}^{*} .
\end{aligned}
$$

Thus, if we invoke (1.2), we see that

$(\partial d(k)+d(k) \partial) \omega=\sum_{j=1}^{N}\left(e_{j}^{*} \partial_{e_{j}} p\right) \otimes d e_{i_{1}}^{*} \wedge \cdots \wedge d e_{i_{l}}^{*}+l \omega+\sum_{\alpha \in R_{+}} k_{\alpha}\left(1 \otimes 1-r_{\alpha} \otimes r_{\alpha}\right) \omega$.

For $m=0,1, \ldots$, let $\mathscr{P}_{m}$ denote the homogeneous polynomials of degree $m$, and put $K_{m}^{l}=\mathscr{P}_{m} \otimes_{\mathbf{C}} \bigwedge_{l} \mathfrak{a}^{*}$. The $K_{m}^{l}$ are invariant under the $G$-action, so there is a further splitting $K_{m}^{l}=\bigoplus_{\tau \in \widehat{G}} K_{m, \tau}^{l}$, where $K_{m, \tau}^{l}$ is the $\tau$-isotypical component in $K_{m}^{l}$. If $\omega \in L_{m, \tau}^{l}$, then $(\partial d(k)+d(k) \partial) \omega=\left(l+m+\lambda_{\tau}(k)\right) \omega$. It follows from Corollary 2.2 in [D3] that $\operatorname{Re}\left(\lambda_{\tau}(k)\right) \geq 0$ if $\operatorname{Re}(k) \geq 0$. Note that $K^{\bullet}$ is a direct sum of finite-dimensional subcomplexes $K^{\bullet}(n)=\bigoplus_{l+m=n} K_{m}^{l}$.

Corollary 3.3. Suppose that $\operatorname{Re}(k) \geq 0, \omega \in K^{l}(l \geq 1)$ and $d(k) \omega=0$. Then $\omega \in d(k) K^{l-1}$.

Proof. By Lemma 3.1 and homogeneity, the statement reduces to the case that $\omega \in K_{m, \tau}^{l}$. But for such $\omega$ the Corollary is obvious from the above remarks.

Corollary 3.4. We have

$$
T_{\xi}(k) T_{\eta}(k)=T_{\eta}(k) T_{\xi}(k) \quad(\xi, \eta \in \mathfrak{a}, k \in K)
$$

in $\operatorname{End}_{\mathbf{C}}(\mathscr{P})$.

Proof. By linearity, it is sufficient to prove this for $\xi=e_{i}$ and $\eta=e_{j}$. Furthermore, as a consequence of the polynomial dependence on $k$, we may assume that $\operatorname{Re}(k) \geq 0$. In the notation of Corollary 2.2 , take $L_{i}=\mathscr{P}_{i}, \partial_{j}=T_{e_{j}}(0)$ and $\delta_{j}=T_{e_{j}}(k)$; the conditions (i) to (iv), (i') and (ii') are then satisfied. We verify (b) in Corollary 2.2. To this end, let $\left\{p_{j}\right\}_{j=1}^{N}$ be a closed 1-form (in the terminology of Corollary 2.2). Consider $\omega=\sum_{j=1}^{N} p_{j} \otimes d e_{j}^{*} \in K^{1}$; then $d(k) \omega=0$. By Corollary 3.3, $\omega$ is exact and we conclude (a) in Corollary 2.2.

As a consequence of Corollary 3.4 we see that $d(k)$ is a boundary operator on $K^{\bullet}$ (this statement is in fact equivalent to Corollary 3.4); hence we may form the cohomology groups $H^{i}\left(K^{\bullet}, d(k)\right)$ of the complex $\left(K^{\bullet}, d(k)\right)$.

Corollary 3.5. The following are equivalent:

1. $k \in K^{\mathrm{reg}}$.

2. There exists a linear isomorphism $V(k)$ of $\mathscr{P}$, homogeneous of degree 0 , such that $\left.V(k)\right|_{\mathscr{P}_{0}}=\mathrm{id}$ and $T_{\xi}(k) V(k)=V(k) \partial_{\xi} \quad \forall \xi \in \mathfrak{a}$.

3. There exists an endomorphism $V(k)$ of $\mathscr{P}$, homogeneous of degree 0 , such that $\left.V(k)\right|_{\mathscr{P}_{0}}=\mathrm{id}$ and $T_{\xi}(k) V(k)=V(k) \partial_{\xi} \quad \forall \xi \in \mathfrak{a}$. 
If $V(k)$ exists, it is uniquely determined by the four properties in 2.

Proof. $1 \Rightarrow 2$. Combine Corollary 3.4 and Corollary 2.2.

$2 \Rightarrow 3$. This is trivial.

$3 \Rightarrow 2$. Using the intertwining property, it is easily seen by induction with respect to the homogeneous degree that $V(k)$ is necessarily injective.

$2 \Rightarrow 1$. This is trivial.

The uniqueness statement is part of Corollary 2.2(d).

Corollary 3.6. The following are equivalent:

1. $H^{i}\left(K^{\bullet}, d(k)\right)=0 \quad \forall i>0$.

2. $\operatorname{dim}_{\mathrm{C}} H^{0}\left(K^{\bullet}, d(k)\right)=1$.

3. $k \in K^{\text {reg }}$.

Proof. This is a consequence of Corollary 3.5 , except for the implication $1 \Rightarrow 2$. But this follows from the fact that the Euler characteristic of $K^{\bullet}(n)$ is zero if $n>0$.

In the case where $k_{\alpha}=k \in \mathbf{C}(\forall \alpha \in R)$ we can give an alternative description for the singular polynomials:

Theorem 3.7. Let $p \in \mathscr{P}_{n, \tau}$ (the $\tau$-isotypical component of $\left.\mathscr{P}_{n}\right)$. Then the following are equivalent:

1. $(\exists k \in \mathbf{C}$ such that $d(k) p=0) \vee(\tau=1)$.

2. $\exists k_{1}, k_{2} \in \mathbf{C}, k_{1} \neq k_{2}$ such that $d\left(k_{1}\right) d\left(k_{2}\right) p=0$.

3. $\forall k_{1}, k_{2} \in \mathbf{C}$ we have $d\left(k_{1}\right) d\left(k_{2}\right) p=0$.

Proof. $1 \Rightarrow 3$. By continuity, it is sufficient to prove this for $k_{1} \neq k$. Then there exists $\alpha \in \mathbf{C}$ such that $k_{2}=\alpha k_{1}+(1-\alpha) k$. Now the result follows from the observation that $d\left(k_{2}\right)=\alpha d\left(k_{1}\right)+(1-\alpha) d(k)$ and the fact that $d^{2}\left(k_{1}\right)=0$ (see Corollary 3.4). If $\tau=1$, then $d\left(k_{2}\right) p=d\left(k_{1}\right) p$.

$3 \Rightarrow 2$. This is trivial.

$2 \Rightarrow 1$. If $d\left(k_{1}\right) d\left(k_{2}\right) p=0$, then $\partial d\left(k_{1}\right) d\left(k_{2}\right) p=0$. Since $\partial p=0$ we have, using Proposition 3.1 and 3.2,

$$
\begin{aligned}
& \left(\partial d\left(k_{1}\right)+d\left(k_{1}\right) \partial\right) d\left(k_{2}\right) p=d\left(k_{1}\right)\left(\partial d\left(k_{2}\right)+d\left(k_{2}\right) \partial\right) p \\
& \quad \Rightarrow\left(\left(m+k_{1} \lambda_{\tau}\right) d\left(k_{2}\right)-\left(m+k_{2} \lambda_{\tau}\right) d\left(k_{1}\right)\right) p=0 \\
& \quad \Rightarrow \tau=1 \vee\left(\tau \neq 1 \wedge d\left(\frac{k_{2}\left(m+k_{1} \lambda_{\tau}\right)-k_{1}\left(m+k_{2} \lambda_{\tau}\right)}{\lambda_{\tau}\left(k_{1}-k_{2}\right)}\right) p=0\right) \\
& \quad \Rightarrow \tau=1 \vee\left(\tau \neq 1 \wedge d\left(-\frac{m}{\lambda_{\tau}}\right) p=0\right) .
\end{aligned}
$$

Corollary 3.8. Let $\left\{e_{1}, \ldots, e_{N}\right\}$ be a basis for a. Put

$$
\Delta_{i}=\sum_{\alpha \in R_{+}}\left(\alpha, e_{i}\right) \frac{1-r_{\alpha}}{\alpha^{*}} \quad(i=1, \ldots, N) .
$$

Then

$$
\begin{aligned}
\{p \in & \left.\mathscr{P}_{+} \mid\left(\partial_{i} \Delta_{j}-\partial_{j} \Delta_{i}\right) p=0 \forall i, j\right\} \\
& =\left\{p \in \mathscr{P}_{+} \mid\left(\Delta_{i} \partial_{j}-\Delta_{j} \partial_{i}\right) p=0 \forall i, j\right\} \\
& =\operatorname{Span}\left(\left\{p \in \mathscr{P}_{+} \mid \exists k \in \mathbf{C} \text { s.t. } T_{\xi}(k) p=0 \forall \xi \in \mathfrak{a}\right\} \cup I_{+}\right) \\
& =S+I_{+},
\end{aligned}
$$


where $I_{+}$denotes the invariant polynomials with zero constant term.

Proof. This follows from Theorem 3.7 if one notes that $\Delta_{i}=T_{e_{i}}(1)-T_{e_{i}}(0)$.

\section{THE SINGULAR SET}

In this section we determine the singular set. A bilinear form on $\mathscr{P}$ (introduced in [D4]), together with the work of Heckman [H] on shift operators, will enable us to reduce the question to the determination of the zero locus of a certain polynomial. This zero locus already occurred in the work of Opdam [O] as the poles of the generalized Bessel function. The results in [loc. cit], combined with the explicit form of a certain Poincare polynomial (introduced and determined by Macdonald), turn out to be sufficient to determine the aforementioned polynomial explicitly in all cases.

As a consequence of the commutativity of the $T_{\xi}(k)$ for fixed $k$, there exists an algebra homomorphism from $\mathscr{P}$ to $\operatorname{End}_{\mathbf{C}}(\mathscr{P})$ which sends $\xi^{*}$ to $T_{\xi}(k)$ and 1 to the identity. If $p \in \mathscr{P}$, we will denote the corresponding operator by $p(T(k))$. We can thus make the following definition:

$$
(p, q)_{k}=(p(T(k)) q)(0)(p, q \in \mathscr{P}) .
$$

Let us collect some basic properties in the following lemma.

Lemma 4.1. $1 .(\cdot, \cdot)_{k}$ is symmetric.

2. $\left(\xi^{*} p, q\right)_{k}=\left(p, T_{\xi}(k) q\right)_{k} \quad(\xi \in \mathfrak{a}, p, q \in \mathscr{P})$.

3. $\left(T_{\xi}(k) p, q\right)_{k}=\left(p, \xi^{*} q\right)_{k} \quad(\xi \in \mathfrak{a}, p, q \in \mathscr{P})$.

4. $(g \cdot p, g \cdot q)_{k}=(p, q)_{k}(g \in G, p, q \in \mathscr{P})$.

5. $\left(\mathscr{P}_{r, \tau}, \mathscr{P}_{s, \sigma}\right)_{k}=0$ if $r \neq s$ or $\tau \neq \sigma$.

Proof. 1. This was proved in [D4].

2. Obvious from the definition.

3. This follows from part 1 and 2.

4. This is a consequence of (1.1).

5. It is obvious that homogeneous polynomials of different degree are orthogonal. The statement concerning the $G$-type follows from 4 and the fact that the characters of the representations of $G$ take the same value on $g$ and $g^{-1}$ for all $g \in G$. This is a consequence of a result of Springer [S], stating that any $g \in G$ can be written as $g=\theta_{1} \theta_{2}$ with $\theta_{1}^{2}=\theta_{2}^{2}=1$, which shows that $g$ and $g^{-1}$ are in the same conjugacy class.

The form $(\cdot, \cdot)_{k}$ can be degenerate. We therefore define

$$
\operatorname{Rad}(k)=\left\{p \in \mathscr{P} \mid(p, q)_{k}=0 \forall q \in \mathscr{P}\right\} .
$$

Remark 4.2. It follows from Lemma 4.1 that $\operatorname{Rad}(k)$ is a graded ideal in $\mathscr{P}$ which is invariant under the $T_{\xi}(k)$ and the $G$-action. If $p \in \operatorname{Rad}(k)$, then $\prod_{g \in G} g \cdot p \in \operatorname{Rad}(k)$; thus, if $\operatorname{Rad}(k) \neq 0, \operatorname{Rad}(k)$ contains a nonzero $G$ invariant and hence all $G$-types. Note that the only constant in $\operatorname{Rad}(k)$ is 0 .

Proposition 4.3. The following are equivalent:

1. $k \in K^{\text {reg }}$.

2. $\operatorname{Rad}(k)=0$. 
Proof. $1 \Rightarrow 2$. If $\operatorname{Rad}(k) \neq 0$, take any nonzero homogeneous $p \in \operatorname{Rad}(k)$ of minimal degree. By the properties of $\operatorname{Rad}(k)$ and the choice of $p, p$ must be annihilated by the $T_{\xi}(k)$, which is a contradiction.

$2 \Rightarrow 1$. This is obvious.

As it turns out, $K^{\text {sing }}$ is invariant under translations over negative integers. An important ingredient for our proof of this fact is the following theorem of Heckman $[\mathrm{H}]$. Let $\mathscr{P} G$ denote the $G$-invariant polynomials, and let $\pi=$ $\prod_{\alpha \in R_{+}} \alpha^{*}$ be the fundamental alternating polynomial. The multiplicity function that is constant with value 1 will be denoted by $1_{R}$.

Theorem 4.4. For all $p, q \in \mathscr{P}^{G}$ we have

$$
p(T(k))(\pi q)=\pi p\left(T\left(k+1_{R}\right)\right)_{q} .
$$

Corollary 4.5. Let $p, q \in \mathscr{P} G$. Then $(\pi p, \pi q)_{k}=(\pi, \pi)_{k}(p, q)_{k+1_{R}}$.

Proof. We may assume that $p$ and $q$ are homogeneous and of equal degree. Then

$$
\begin{aligned}
(\pi p, \pi q)_{k} & =(\pi, p(T(k)) \pi q)_{k}=\left(\pi, \pi p\left(T\left(k+1_{R}\right)\right) q\right)_{k} \\
& =\left(\pi(T(k)) \pi, p\left(T\left(k+1_{R}\right)\right) q\right)_{k} .
\end{aligned}
$$

Now both arguments in the right-hand side are constants, so the pairing is just the product of these constants.

Corollary 4.6. If $k+1_{R} \in K^{\text {sing }}$, then $k \in K^{\text {sing }}$.

Proof. If $k+1_{R} \in K^{\text {sing }}$, then $\operatorname{Rad}\left(k+1_{R}\right) \neq 0$ by Proposition 4.3. By Remark 4.2, we see that $\operatorname{Rad}\left(k+1_{R}\right)$ contains an invariant nonzero $q$, homogeneous of some degree $n$. From Corollary 4.5 , we infer that $(\pi p, \pi q)_{k}=0$ for all $p \in \mathscr{P} G$, homogeneous of degree $n$. But then $\pi q$ is orthogonal to the isotypical component (in degree $n+\left|R_{+}\right|$) corresponding to the determinant representation of $G$. Thus, as a consequence of Lemma 4.1.5, $\pi q \in \operatorname{Rad}(k)$ and $k \in K^{\text {sing }}$.

Lemma 4.7. Let $k \in K^{\text {sing }}$ and put $n_{0}=\max _{n \in N}\left\{n \mid k+n \cdot 1_{R} \in K^{\text {sing }}\right\}$. Then $(\pi, \pi)_{k+n_{0} \cdot 1_{R}}=0$.

Proof. Recall that $k \in K^{\mathrm{reg}}$ as soon as $\operatorname{Re}(k) \geq 0$, so $n_{0}$ is finite. Since $k+n_{0} \cdot 1_{R} \in K^{\text {sing }}$, we see by Remark 4.2 that there exists a nonzero $q \in \mathscr{P} G$ such that $(\pi p, \pi q)_{k+n_{0} \cdot 1_{R}}=0$ for all $p \in \mathscr{P} G$. Thus, by Corollary 4.5 we have $(\pi, \pi)_{k+n_{0} \cdot 1_{R}}(p, q)_{k+\left(n_{0}+1\right) \cdot 1_{R}}=0$ for all $p \in \mathscr{P} G$. Now $(p, q)_{k+\left(n_{0}+1\right) \cdot 1_{R}}$ cannot be zero for all $p \in \mathscr{P}^{G}$. For, if this were the case, then

$$
q \in \operatorname{Rad}\left(k+\left(n_{0}+1\right) \cdot 1_{R}\right)
$$

by Lemma 4.1.5 and consequently $k+\left(n_{0}+1\right) \cdot 1_{R} \in K^{\text {sing }}$ by Proposition 4.3, contradicting the definition of $n_{0}$. Thus $(\pi, \pi)_{k+n_{0} \cdot 1_{R}}=0$.

Theorem 4.8. Let $K^{0}=\left\{k \in K \mid(\pi, \pi)_{k}=0\right\}$. Then $K^{\text {sing }}=\bigcup_{n=0}^{\infty}\left\{K^{0}-n \cdot 1_{R}\right\}$. Proof. From Lemma 4.7 we have " $\subset$ ". For the reverse inclusion, note that if $(\pi, \pi)_{k}=0$, then $\pi \in \operatorname{Rad}(k)$ (for reasons of $G$-type) so $k \in K^{\text {sing }}$. Thus " $\supset$ " is a consequence of Corollary 4.6.

The sets $K^{\text {sing }}$ and $K^{0}$ already occurred in [O], where they were denoted by $Z$ and $Z_{B}$. In fact, the description of $K^{\text {sing }}$ in Theorem 4.8 and the results in 
[loc. cit, §9] show "by inspection" that $K^{\text {sing }}$ is the pole set of the generalized Bessel function. There is also a more direct way to see that this must be the case. To this end, fix $k \in K$ and consider the problem of finding a family of $G$-invariant formal power series $\left\{J_{G}(\lambda, k, \xi) \mid \lambda \in \mathfrak{a}_{\mathbf{C}}\right\}$ in $\xi$ such that

$$
\left\{\begin{aligned}
p(T(k)) J_{G}(\lambda, k, \cdot) & =p(\lambda) J_{G}(\lambda, k, \cdot) & & \left(\forall \lambda \in \mathfrak{a}_{\mathbf{C}}, \forall p \in \mathscr{P}^{G}\right), \\
J_{G}(\lambda, k, 0) & =1 & & \left(\forall \lambda \in \mathfrak{a}_{\mathbf{C}}\right) .
\end{aligned}\right.
$$

Evaluating at zero, a solution of (4.1) apparently has the following reproducing property:

$$
\left(J_{G}(\lambda, k, \cdot), p\right)_{k}=p(\lambda) \quad\left(\forall \lambda \in \mathfrak{a}_{\mathbf{C}}, \forall p \in \mathscr{P}^{G}\right) .
$$

From this one sees that one must have $k \in K^{\text {reg }}$ and that $J_{G}(\lambda, k, \cdot)$ is then uniquely determined for all $\lambda$, hence equal to $V(k) \frac{1}{|G|} \sum_{g \in G} \exp (g \lambda, \cdot)$. On the other hand, if $k \in K^{\text {reg }}$ then $V(k) \frac{1}{|G|} \sum_{g \in G} \exp (g \lambda, \cdot)$ satisfies (4.1). Hence (4.1) has a formal solution if and only if $k \in K^{\text {reg }}$ and this solution is then equal to $V(k) \frac{1}{|G|} \sum_{g \in G} \exp (g \lambda, \cdot)$. This family of formal power series is in fact meromorphic on $a_{C} \times K \times a_{C}$; the poles occur exactly when the restriction of $V(k)$ to the $G$-invariants has a pole, i.e., in $K^{\text {sing }}$. But $(4.1)$ is just the defining equation for the generalized Bessel function, so $K^{\text {sing }}$ and the pole set of the generalized Bessel function coincide as claimed.

In view of Theorem 4.8, all that remains to be done is the determination of $K^{0}$. If $R$ is reducible, then one sees easily that $(\pi, \pi)_{k}$ factors as the product of the corresponding polynomials for the irreducible components of $R$. Hence we may assume that $R$ is irreducible, implying that $K$ is at most two-dimensional.

We recall some definitions and results from [O].

Let $B \in \mathbf{C}[K]$ be defined by $B(k)=(\pi, \pi)_{k}$ and introduce the polynomial $b$ in one variable by $b(s)=B\left(s 1_{R}\right)$. Then (see [loc. cit.]) $b$ has degree $\left|R_{+}\right|$. Let $R=\bigcup_{i=1}^{m} C_{i}$ be the decomposition of $R$ into minimal $G$-orbits. For $g \in G$, let $l_{i}(g)$ be the number of simple reflections $r_{\alpha}$ with $\alpha \in C_{i}$ that occur in a minimal expression for $g$ (this is well defined). For $k=\sum_{i=1}^{m} k_{i} 1_{C_{i}} \in K$, put $k^{l}(g)=\prod_{i=1}^{m} k_{i}^{l_{i}(g)}$, and let $P_{G}(k)=\sum_{g \in G} k^{l}(g)$ be the Poincaré polynomial of $G$. These polynomials were introduced and determined by Macdonald [M]. If one takes the $k_{i}$ all equal, they reduce to the Poincare polynomial of the coinvariant algebra of $G$. From the results in $[\mathrm{O}]$ we extract the following.

Theorem 4.9. 1. $K^{\text {sing }} \subset K^{P}$, where $K^{P}$ is the zero-locus of $k \mapsto P_{G}(\exp (2 \pi i k))$ and $\exp (2 \pi i k)$ is to be calculated componentwise. $G$.

2. $b(k)=|G| \prod_{i=1}^{n} \prod_{j=1}^{d_{i}-1}\left(d_{i} k+j\right)$, where the $d_{i}$ are the primitive degrees of

If $m=1$, Theorem 4.9.2 answers our question. So let us assume that $m=2$. It follows from Macdonald's list that $K^{P}$ consists of a countable union of lines. Since in particular $K^{0} \subset K^{P}$, the following lemma allows us to conclude that $B$ must be a product of these lines.

Lemma 4.10. Let $L$ be an algebraically closed uncountable field. Let $F \in$ $L[X, Y]$ be nonconstant and suppose that $V(F) \subset \bigcup_{i=1}^{\infty} V\left(F_{i}\right)$ where the $F_{i}$ are irreducible (here $V(P)$ denotes the algebraic set determined by $P$ ). Then $F$ is the product of a finite number of the $F_{i}$, up to a constant. 
Proof. We use results of $\S 1.6$ in [Fu]. First of all, we may assume that $F$ is irreducible. Since $V(F)=\bigcup_{i=1}^{\infty}\left(V(F) \cap V\left(F_{i}\right)\right)$ and $V(F)$ is uncountable, there is $F_{i_{0}}$ such that $V(F) \cap V\left(F_{i_{0}}\right)$ is infinite. Hence at least one of the irreducible components of $V(F) \cap V\left(F_{i_{0}}\right)$ is an irreducible curve $V(C)$. But then it is a well-known consequence of the Nullstellensatz that $F$ and $C$ must be multiples of each other. Since the same argument applies to $F_{i_{0}}$ and $C$, the proof is finished.

Thus $B$ splits as a product of linear factors, to be taken from the lines constituting $K^{P}$. Now inspection of Macdonald's list shows that each of these lines is of the form $p k_{1}+q k_{2}+r$ where $p$ and $q$ are both real (in fact integer) and nonnegative. This implies that $B$ factors as a product of exactly $R_{+}$(the degree of $b$ ) linear factors, each of which (by Theorem 4.9.2) intersects $C .1_{R}$ in $(-1,0)$.

Let us show how we can now determine $B$ for the dihedral groups $I_{2}(2 m)$ of order $4 \mathrm{~m}$. There are $2 \mathrm{~m}$ positive roots. From Macdonald's list we have $P_{G}\left(t_{1}, t_{2}\right)=\left(1+t_{1}\right)\left(1+t_{2}\right)\left(1-u^{m}\right) /(1-u)$ where $u=t_{1} t_{2}$. It is then easy to see that $K^{P}$ consists of three sets of lines:

1. $k_{1}=\frac{1}{2}+l(l \in \mathbf{Z})$.

2. $k_{2}=\frac{1}{2}+l(l \in \mathbf{Z})$.

3. $k_{1}+k_{2}=\frac{l}{m}(l \in \mathbf{Z}, m \nmid l)$.

By inspection, there are only $2 m$ lines that intersect C. $1_{R}$ in $(-1,0)$, namely $k_{1}+\frac{1}{2}, k+\frac{1}{2}$, and $k_{1}+k_{2}+\frac{j}{m}(j=1, \ldots, m-1, m+1, \ldots, 2 m-1)$. So $B$ is known up to a constant, which then follows from Theorem 4.9.2. This method also works for $B_{n}$ and $F_{4}$. We summarize the results in the following theorem.

Theorem 4.11. Let $R$ be irreducible. If there is only one $G$-orbit in $R$, then

$$
(\pi, \pi)_{k}=|G| \prod_{i=1}^{n} \prod_{j=1}^{d_{i}-1}\left(d_{i} k+j\right),
$$

where the $d_{i}$ are the primitive degrees of $G$. In the remaining cases $(\pi, \pi)_{k}$ is equal to

$$
2^{\frac{1}{2} n(n+1)} n !\left\{\prod_{j=0}^{n-1} \prod_{l=1}^{j+1}\left(2 j k_{1}+2 k_{2}+2 l-1\right)\right\} \cdot\left\{\prod_{j=1}^{n-1} \prod_{l=1}^{j}\left((j+1) k_{1}+l\right)\right\},
$$

where $k_{1}$ corresponds to the long root in the crystallographic root system.

$F_{4}$ :

$$
\begin{aligned}
2^{20} 3^{4} & \cdot \prod_{i=1,2}\left\{\left(2 k_{i}+1\right)\left(3 k_{i}+1\right)\left(3 k_{i}+2\right)\left(2 k_{i}+2 u+1\right)\right. \\
& \left.\cdot\left(2 k_{i}+2 u+3\right)\left(2 k_{i}+2 u+5\right)\right\} \\
& \cdot(6 u+1)(6 u+5)(6 u+7)(6 u+11)
\end{aligned}
$$

where $u=k_{1}+k_{2}$. 
$I_{2}(2 m)$ :

$$
4 m^{2}\left(2 k_{1}+1\right)\left(2 k_{2}+1\right) \prod_{\substack{j=1 \\ j \neq m}}^{2 m-1}\left(m\left(k_{1}+k_{2}\right)+j\right)
$$

\section{SiNGULAR POLYNOMIALS: ONE-DIMENSIONAL REPRESENTATIONS AND THE DIHEDRAL GROUPS}

The singular set was determined in the previous section for arbitrary $G$. In general, the set of singular polynomials (as in the introduction) is still unknown. We can, however, determine the singular polynomials completely if $G$ is a dihedral group. For general $G$, we are able to give a description of the singular polynomials that transform according to a one-dimensional representation of $G$.

We recall the definition of the $k$-Laplacian:

$$
\Delta(k)=\sum_{i=1}^{N} T_{\xi_{i}}^{2}(k),
$$

where $\left\{\xi_{1}, \ldots, \xi_{N}\right\}$ is any orthonormal basis for a. If $f \in \mathscr{P}_{n}$ and $k \in K$, we say that $f$ has a $k$-Laplace decomposition if $f$ can be written as

$$
f=\sum_{j \geq 0}|x|^{2 j} f_{n-2 j}
$$

with $f_{n-2 j} \in \mathscr{P}_{n-2 j}$ and $\Delta(k) f_{n-2 j}=0$ for all $j$. We will investigate the values of $k$ sufficient for existence and uniqueness of decompositions of this kind. The result will be helpful in the dihedral case. Put $\gamma=\sum_{\alpha \in R_{+}} k_{\alpha}$. Suppose that $\tau \in \widehat{G}$ and that $\tau$ is realized on $V_{n} \subset \mathscr{P}_{n}$. Let $n_{0}=\min _{j \in \mathbf{N}}\{n-2 j \mid \tau$ occurs in $\left.\mathscr{P}_{n-2 j}\right\}$. If $n=n_{0}$, then $\Delta(k) V_{n}=(0)$, and the decomposition is trivial (but unique).

Lemma 5.1. Each $f \in V_{n}$ has a unique $k$-Laplace decomposition if $-\left(\frac{N}{2}+\gamma\right) \neq$ $n_{0}, n_{0}+1, n_{0}+2, \ldots, n-2$.

Proof. By Theorem 1.11 in [D1], we have the decomposition

$$
f=\sum_{j \leq n / 2}|x|^{2 j} f_{n-2 j},
$$

valid for generic values of $k$, with

$$
\begin{aligned}
f_{n-2 j}= & \left(4^{j} j !\left(\frac{N}{2}+\gamma+n-2 j\right)_{j}\right)^{-1} \\
& \cdot \sum_{i \leq[n / 2]-j}\left(4^{i} i !\left(-\frac{N}{2}-\gamma-n+2 j+2\right)_{i}\right)^{-1}|x|^{2 i} \Delta(k)^{i+j} f
\end{aligned}
$$

and $\Delta(k) f_{n-2 j}=0$. The verification is based on the identity

$$
\sum_{j=0}^{l} \frac{1}{j !(l-j) !(A-2 j)_{j}(-A+2 j+2)_{l-j}}=0
$$


for $l=1,2, \ldots$. There are poles at $A=2,3, \ldots, 2 l$. The sum appears as the coefficient of $\Delta(k)^{l} f$, hence it must be valid for $n-2 l \geq n_{0}$, that is $l \leq \frac{\left(n-n_{0}\right)}{2}$. This occurs if $\frac{N}{2}+\gamma+n$ avoids $2,3,4, \ldots, n-n_{0}$, as claimed. (The effect of the forbidden values is to allow the situation $\Delta(k)\left(|x|^{2 j} f_{n-2 j}\right)=0$ and $\Delta(k) f_{n-2 j}=0$, resulting in "too many" harmonics of degree $n$.)

The one-dimensional representations. Suppose $G$ has two orbits $C_{1}$ and $C_{2}$ in $R$ and $k$ has the values $k_{1}$ and $k_{2}$, respectively. There is an alternating polynomial for each class, namely $p_{i}=\prod_{\alpha \in C_{i}, \alpha \in R_{+}} \alpha^{*}$. For $\alpha \in R$ we have $r_{\alpha} p_{i}=-p_{i}$ if $\alpha \in C_{i}$ and $r_{\alpha} p_{i}=p_{i}$ otherwise. The present discussion specializes easily to the case that $G$ has only one orbit in $R$.

Recall the definition of the $k$-gradient:

$$
\nabla(k) f=\nabla f+\sum_{\alpha \in R_{+}} k_{\alpha}\left(\frac{1-r_{\alpha}}{\alpha^{*}} f\right) \alpha .
$$

Obviously, $f$ is singular if and only if $\nabla(k) f=0$ for some $k$.

Proposition 5.2. The singular polynomials of the isotypes of the nontrivial one dimensional representations of $G$ are $p_{1}^{2 n_{1}+1}$ (if $k_{1}=-n_{1}-\frac{1}{2}$ ), $p_{2}^{2 n_{2}+1}$ (if $k_{2}=-n_{2}-\frac{1}{2}$ ) and $p_{1}^{2 n_{1}+1} p_{2}^{2 n_{2}+1}$ (if $k_{1}=-n_{1}-\frac{1}{2}$ and $k_{2}=-n_{2}-\frac{1}{2}$ ) for arbitrary $n_{1}, n_{2}=0,1,2, \ldots$.

Proof. The general function of the same isotype as $p_{1} p_{2}$ is $p_{1} p_{2} g$ where $g$ is invariant. Then

$$
\nabla(k)\left(p_{1} p_{2} g\right)=p_{1} p_{2} \nabla g+p_{1} p_{2} g(1+2 k) \sum_{\substack{\alpha \in C_{1} \\ \alpha \in R_{+}}} \frac{\alpha}{\alpha^{*}}+p_{1} p_{2} g\left(1+2 k_{2}\right) \sum_{\substack{\alpha \in C_{2} \\ \alpha \in R_{+}}} \frac{\alpha}{\alpha^{*}} .
$$

For $p_{1} p_{2} g$ to be singular, $g$ must satisfy

$$
\frac{\nabla g}{g}=-\left(1+2 k_{1}\right) \sum_{\substack{\alpha \in C_{1} \\ \alpha \in R_{+}}} \frac{\alpha}{\alpha^{*}}-\left(1+2 k_{2}\right) \sum_{\substack{\alpha \in C_{2} \\ \alpha \in R_{+}}} \frac{\alpha}{\alpha^{*}} .
$$

Thus, $g$ is a scalar multiple of $\left|p_{1}\right|^{-\left(1+2 k_{1}\right)}\left|p_{2}\right|^{-\left(1+2 k_{2}\right)}$. For this to be an invariant polynomial we must have $-1-2 k_{1}=2 n_{1},-1-2 k_{2}=2 n_{2}$ for nonnegative integers $n_{1}, n_{2}$. The other cases $p_{1}^{2 n_{1}+1}, p_{2}^{2 n_{2}+1}$ are shown similarly.

The dihedral groups. The dihedral groups $I_{2}(m)$ act on $\mathbf{R}^{2} \simeq \mathbf{C}$. Let $\omega=$ $\exp (2 \pi i / m)$, then the group $G$ consists of the reflections $z \mapsto \bar{z} \omega^{j}$ and the rotations $z \mapsto z \omega^{j} \quad(0 \leq j \leq m-1, z \in \mathbf{C})$. $G$ has one conjugacy class of reflections when $m$ is odd, and two classes when $m$ is even. When $m$ is odd, we use $k$ to denote the value of the parameter and write $T(k), \bar{T}(k)$ for the operators of Definition 3.1 in [D2]. The singular polynomials $f$ are those for which $T(k) f=\bar{T}(k) f=0$. In [D2] we already determined the solutions of $\bar{T}(k) f=0$, for any $k$. Essentially we only have to apply the condition $T(k) f=0$.

The dihedral groups, odd case. Let $m$ be odd $(m \geq 3) . \quad \operatorname{Span}\left(\left\{z^{l}, \bar{z}^{l}\right\}\right)$ and $\operatorname{Span}\left(\left\{z^{m-l}, \bar{z}^{m-l}\right\}\right)$ are of the same isotype for $1 \leq l \leq m-1$ (and this includes all two-dimensional representations of $G)$. For each such $l$ and 
each $n=0,1,2, \ldots$, there is a unique (up to scalar multiplication) polynomial of isotype $l$, degree $n m+l$, in the kernel of $\bar{T}(k)$, namely $f_{n m+l}(z)=$ $z^{l} C_{n}^{(k, k+1)}\left(z^{m}\right)$ (see [D2, p. 181]). Here $C_{n}^{(\delta, \varepsilon)}(z)$ is the Heisenberg polynomial defined by

$$
C_{n}^{(\delta, \varepsilon)}(z)=\sum_{j=0}^{n} \frac{(\delta)_{j}(\varepsilon)_{n-j}}{j !(n-j) !} \bar{z}^{j} z^{n-j} \quad(z \in \mathbf{C}),
$$

generated by

$$
(1-t \bar{z})^{-\delta}(1-t z)^{-\varepsilon}=\sum_{n=0}^{\infty} C_{n}^{(\delta, \varepsilon)}(z) t^{n} .
$$

The polynomial $C_{n}^{(\delta, \varepsilon)}$ is nonzero, unless $\delta=-n_{1}, \varepsilon=-n_{2}$ with $n_{1}, n_{2}=$ $0,1,2, \ldots$ and $n>n_{1}+n_{2}$. Now $T(k) f_{n m+l}(z)=(l+m n+m k) f_{n m+l-1}(z)$. Setting $k=-n-l / m$ gives the singular polynomial $\left(k \notin \mathbf{Z}\right.$, so $C_{n}^{(k, k+1)}\left(z^{m}\right)$ is not zero). The complex conjugate of $f_{n m+l}$ is the other basis element for the singular polynomials of degree $n m+l$. There are no other singular polynomials of this isotype and degree. They would be harmonic, since $\Delta(k)=4 T(k) \bar{T}(k)$, but by Lemma 5.1 there are only two linearly independent harmonic polynomials provided $-(1+m k)$ avoids $n_{0}, n_{0}+1, \ldots, n m+l-2$, and of course $-1-m k=-1+m n+l$.

By Lemma 5.1 the alternating polynomial $p(z)=z^{m}-\bar{z}^{m}$, gives rise to the singular polynomials $\left(z^{m}-\bar{z}^{m}\right)^{2 n+1}$ with $k=-\frac{1}{2}-n$.

This is the entire list of singular polynomials because the only other representation of $G$ is the trivial one.

The dihedral groups, even case. Let $m \geq 2$ be even and let $G=I_{2}(2 m)$. Put $\omega=\exp (\pi i / m)$. Associate the parameter values $k_{1}$ to the reflections $z \mapsto \bar{z} \omega^{2 j}$ and $k_{2}$ to $z \mapsto \bar{z} \omega^{2 j+1}(0 \leq j \leq m-1)$. We will write $\Delta\left(k_{1}, k_{2}\right), T\left(k_{1}, k_{2}\right)$ and $\bar{T}\left(k_{1}, k_{2}\right)$ for the operators. For each $l \in\{1,2, \ldots, m-1\}$ there is an isotype realized on $\operatorname{span}\left\{z^{l}, \bar{z}^{l}\right\}$ and on $\operatorname{span}\left\{z^{2 m-l}, \bar{z}^{2 m-l}\right\}$.

The polynomials of degree $s m+l(1 \leq l \leq m-1, s=0,1,2, \ldots)$ annihilated by $\bar{T}\left(k_{1}, k_{2}\right)$ are (see 3.19 in [D2]) $z^{l} f_{s}\left(z^{m}\right)$ where

$$
\begin{aligned}
f_{2 n}\left(r e^{i \theta}\right)= & r^{2 n} P^{\left(k_{1}-\frac{1}{2}, k_{2}-\frac{1}{2}\right)}(\cos 2 \theta)+\left(\frac{i}{2}\right) r^{2 n}(\sin 2 \theta) P_{n-1}^{\left(k_{1}+\frac{1}{2}, k_{2}+\frac{1}{2}\right)}(\cos 2 \theta), \\
f_{2 n+1}\left(r e^{i \theta}\right)= & r^{2 n}\left(\left(n+k_{1}+\frac{1}{2}\right)(r \cos \theta) P_{n}^{\left(k_{1}-\frac{1}{2}, k_{2}+\frac{1}{2}\right)}(\cos 2 \theta)\right. \\
& \left.+i\left(n+k_{2}+\frac{1}{2}\right)(r \sin \theta) P_{n}^{\left(k_{1}+\frac{1}{2}, k_{2}-\frac{1}{2}\right)}(\cos 2 \theta)\right) \\
& (r \geq 0,0 \leq \theta<2 \pi, n=0,1,2, \ldots) .
\end{aligned}
$$

The Jacobi polynomial is

$$
P_{n}^{(\delta, \varepsilon)}(t)=\frac{(\delta+1)_{n}}{n !}{ }_{2} F_{1}\left(\begin{array}{c}
-n, n+\delta+\varepsilon+1 \\
\delta+1
\end{array} ; \frac{1-t}{2}\right)
$$

there are no poles (parameter values) and the polynomial is of degree $n$, provided $(n+\delta+\varepsilon+1)_{n} \neq 0$. This condition applies in the present case. By the formula in [D2, p. 183] we have

$$
T\left(k_{1}, k_{2}\right) z^{l} f_{s}\left(z^{m}\right)=\left(l+m s+m\left(k_{1}+k_{2}\right)\right) z^{l-1} f_{s}\left(z^{m}\right) .
$$


Thus for $k_{1}+k_{2}=-s-l / m, z^{l} f_{s}\left(z^{m}\right)$ (and its complex conjugate) is a singular polynomial. The parameters $(\delta, \varepsilon)$ in the Jacobi polynomials in $f_{2 n}, f_{2 n+1}$ satisfy $n+\gamma+\delta+1=n+k_{1}+k_{2}+s$ with $s=0,1$ or 2 , and $k_{1}+k_{2} \notin \mathbf{Z}$.

There are no other singular (or harmonic) polynomials for these parameter values and this degree. By Lemma 5.1, $-\left(1+m\left(k_{1}+k_{2}\right)\right)$ must avoid $n_{0}, n_{0}+$ $1, \ldots, m s+l-2$ and $-\left(1+m\left(k_{1}+k_{2}\right)\right)=m s+l-1$. The singular polynomials of alternating isotype are $\left(z^{m}-\bar{z}^{m}\right)^{2 n_{1}+1},\left(z^{m}+\bar{z}^{m}\right)^{2 n_{2}+1}$ and the product of these two, with $k_{1}=-n_{1}-\frac{1}{2}$ for the first, $k_{2}=-n_{2}-\frac{1}{2}$ for the second and both conditions for the product. Lemma 5.1 does not apply in the product case. Here $-1-m\left(k_{1}+k_{2}\right)$ must avoid $2 m, 2 m+1, \ldots, 2 m\left(n_{1}+n_{2}+1\right)-2$ but here $k_{1}+k_{2}=-n_{1}-n_{2}-1$ and $m\left(n_{1}+n_{2}+1\right)$ appears in the list if $n_{1}+n_{2} \geq 2$. Again we have described all the singular polynomials.

\section{EstimATES FOR $\operatorname{dim}_{\mathbf{C}}\left(S_{m, \tau}\right)$ IF $k_{\alpha}=k \forall \alpha \in R$}

Recall the following constructions from [D5]. Let $\mathfrak{h}=\mathfrak{a} \otimes_{\mathbf{R}} \mathbf{C}$. The form $(\cdot, \cdot)$ extends to a bilinear form on $\mathfrak{h}$, which will again be denoted by $(\cdot, \cdot)$. Any $\xi \in \mathfrak{h}$ gives rise, via this form, to $\xi^{*} \in \mathfrak{h}^{*}$ as in the Introduction. Put $\mathfrak{h}^{\text {reg }}=\left\{\xi \in \mathfrak{h} \mid \alpha^{*}(\xi) \neq 0 \forall \alpha \in R\right\}$. Let $\mathscr{O}\left(\mathfrak{h}^{\text {reg }}\right)$ denote the holomorphic functions on $\mathfrak{h}^{\text {reg }}$. Let $\tau \in \widehat{G}$, and let $V_{\tau}$ be the corresponding representation space of dimension $d_{\tau}$. Define the following connection $\nabla^{\tau}(k)$ on $\mathscr{O}\left(h^{\text {reg }}\right) \otimes V_{\tau}$ (the holomorphic sections in the trivial bundle $\mathfrak{h}^{\text {reg }} \times V_{\tau}$ ):

$$
\nabla_{\xi}^{\tau}(k)(f \otimes v)=\left(\partial_{\xi} f\right) \otimes v+k \sum_{a \in R_{+}}(\alpha, \xi)\left(\frac{f}{\alpha^{*}}\right) \otimes\left(1-\tau\left(r_{\alpha}\right)\right) v .
$$

This connection is integrable and has regular singularities.

Let $\rho$ denote the natural representation of $G$ on $\mathscr{O}\left(h^{\text {reg }}\right)$ :

$$
(g \cdot f)(z)=f\left(g^{-1} z\right) \quad\left(g \in G, f \in \mathscr{O}\left(\mathfrak{h}^{\text {reg }}\right), z \in \mathfrak{h}^{\text {reg }}\right) .
$$

Then $\nabla^{\tau}(k)$ is equivariant for $\rho \otimes \tau$ in the sense that

$$
\nabla_{\xi}^{\tau}(k)(\rho(g) f \otimes \tau(g) v)=(\rho(g) \otimes \tau(g))\left(\nabla_{g^{-1} \xi}^{\tau}(k)(f \otimes v)\right) .
$$

Hence we can view $\nabla^{\tau}(k)$ as the inverse image of a connection $\overline{\nabla^{\tau}(k)}$ on $\overline{\mathscr{V}}_{\tau}=\mathscr{O}\left(G \backslash \mathfrak{h}^{\text {reg }}\right) \otimes_{\mathbf{C}} \bar{V}_{\tau}$, where $\bar{V}_{\tau}$ is the local system on $X=G \backslash \mathfrak{h}^{\text {reg }}$ defined by $\bar{V}_{\tau}=\mathfrak{h}^{\text {reg }} \times{ }_{G} V_{\tau}$. The fundamental group of $X$ is the braid group of $G$ (see [B]). The monodromy representation of $\overline{\nabla_{\tau}(k)}$ factorizes through the Hecke algebra $H_{G}(q(k))$ where $q(k)=\exp (-2 \pi i k)$, and where we use the convention that the generators of $H_{G}(q)$ satisfy $\left(T_{i}-1\right)\left(T_{i}+q\right)=0$. The resulting representation of $H_{G}(q)$ is called $Y_{\tau}(k)$. From the construction it is clear that $Y_{\tau}$ is defined over the ring $\mathscr{E}$ of entire functions on $K \cong \mathbf{C}$. By deformation theoretical arguments it was shown in [D5] that $Y_{\tau}(k)$ is irreducible and principal when $\Psi_{t}\left(q^{-1}\right)=\frac{q^{\left|R_{+}\right|} P_{G}\left(q^{-1}\right)}{d_{r}\left(q^{-1}\right)} \neq 0$ (here $d_{\tau}$ is the generic degree of $\tau$ and $P_{G}$ is the Poincaré polynomial of the coinvariant algebra of $\left.G\right)$. Hence loss of irreducibility of some $Y_{\tau}(k)$ can occur only if $P_{G}\left((q(k))^{-1}\right)=0$. Consequently, the zeros of $P_{G}\left(q^{-1}\right)$ correspond to nonsemisimple specializations of $H_{G}$ (see [GU, Y]). Also observe that the trivial representation is never principal if $H_{G}(q)$ is not semisimple. 
We will now apply results of the theory of algebras defined over local domains that are complete. Let $k_{0} \in K \cong \mathbf{C}$, and write $x=k-k_{0}$. Let $\mathscr{R}=\mathbf{C}\{x\}$. (It is important that $\mathscr{R}$ is a complete local principal ideal domain for these statements and this is the reason that we assume $k_{\alpha}=k \forall \alpha$ ). Let $\mathscr{K}=\mathbf{C}\{\{x\}\}=\left\{\sum_{k \geq N} c_{k} x^{k} \mid N \in \mathbf{Z}, c_{k} \in \mathbf{C}\right\}$ be its quotient field. Define $X_{\tau}=\mathbf{R} \otimes_{\varepsilon} Y_{\tau}$ and $A=\mathscr{R} \otimes_{\mathbf{C}\left[q, q^{-1}\right]} H_{G}$. Finally put $\bar{X}_{\tau}=X_{\tau} /(x) \mathscr{R}$ (a module for $\left.A /(x) \mathscr{R}=\bar{A}=H_{G}\left(q\left(k_{0}\right)\right)\right)$, and $\left(X_{\tau}\right)_{\mathscr{K}}=X_{\tau} \otimes_{\mathscr{R}} \mathscr{K}$ (a module for $\left.A_{\mathscr{K}}=\mathscr{K} \otimes_{\mathscr{R}} A=\mathscr{K} \otimes_{\mathrm{C}\left[q, q^{-1}\right]} H_{G}\right)$. Clearly $\left\{\left(X_{\tau}\right)_{\mathscr{K}}\right\}_{\tau \in \widehat{G}}$ is a complete set of representatives for the irreducibles of $A_{\mathscr{K}}$. We are now in the situation as described on p. 67 in [Fe].

Definition 6.1. Let triv be the one-dimensional representation of $H_{G}(q)$ that is defined by sending the $T_{i}$ to 1 . The multiplicity of triv $\in(\bar{A})_{\text {irr }}$ in a decomposition series of $\bar{X}_{\tau}$ will be denoted by $\delta_{\tau}\left(q_{0}\right)$. The multiplicity of triv in $\bar{X}_{\tau}$ as a submodule (i.e., the multiplicity of triv in the socle of $\bar{X}_{\tau}$ ) will be denoted by $\Delta_{\tau}\left(k_{0}\right)$.

This definition makes sense because by [Fe, Theorem 17.7] the number $\delta_{\tau}\left(q_{0}\right)$ depends on $\tau$ and $q_{0}$ only. In fact, these decomposition numbers [Fe, p. 67] have a different interpretation in terms of representation theory of $A$ by virtue of a theorem of Brauer. In our situation we have a one-to-one correspondence between principal indecomposable modules of $A$ and $\bar{A}=H_{G}\left(q_{0}\right)$ (since [Fe, Theorem 13.7] applies). Let $U$ denote the principal indecomposable module of $A$ that corresponds to triv $\in(A)_{\text {irr }}$ (hence $\bar{U}$ is principal indecomposable for $\bar{A}=H_{G}\left(q_{0}\right)$ and $U / \operatorname{rad}(U)=\bar{U} / \operatorname{rad}(\bar{U})=$ triv $)$. We have

Theorem 6.2 (Brauer, see [Fe, Theorem 17.8]).

$$
U_{\mathscr{K}} \cong \bigoplus_{\tau \in \widehat{G}} \delta_{\tau}\left(q_{0}\right)\left(X_{\tau}\right)_{\mathscr{K}}
$$

Corollary 6.3. If $k_{0}$ is such that $H_{G}\left(q_{0}\right)$ is not semisimple, then there exists at least one $\tau \in \widehat{G}, \tau \neq$ triv such that $\delta_{\tau}\left(q_{0}\right) \neq 0$.

Proof. Suppose that $\delta_{\tau}\left(q_{0}\right)=0$ if $\tau \neq$ triv. Clearly $\delta_{\text {triv }}\left(q_{0}\right)=1$ and by Theorem 6.2 we obtain that $U_{\mathscr{K}} \cong(\text { triv })_{\mathscr{K}}$. This implies that $U=t r i v$, and we thus conclude that triv is principal. This contradicts the results of Yamane in $[\mathrm{Y}]$.

Remarks 6.4. 1. $\delta_{\tau}\left(q_{0}\right)=0$ if $\Psi_{\tau}\left(q_{0}^{-1}\right) \neq 0$, since Yamane proved that $\Psi_{\tau}\left(q_{0}^{-1}\right) \neq 0$ implies that $\bar{X}_{\tau}$ is principal and irreducible.

2. Of course $\Delta_{\tau}\left(k_{0}\right) \leq \delta_{\tau}\left(q_{0}\right)$ and $\Delta_{\tau}\left(k_{0}\right)=0$ if $k_{0}>0$.

Proposition 6.5. Assume $\tau \neq$ triv and $k_{0} \notin \frac{1}{2} \mathbf{N}$. Then $\operatorname{dim}_{\mathbf{C}} S_{\tau}\left(k_{0}\right)=d_{\tau} \Delta_{\tau}\left(k_{0}\right)$. Proof. Choose an explicit unitary matrix realization $M_{\tau}$ of $\tau$ on $\mathbf{C}^{d_{\tau}}$ (where $d_{\tau}$ is the dimension of the representation space of $\tau$ ), and let $\left(\overline{\mathscr{V} \tau}, \overline{\nabla^{\tau}}\left(k_{0}\right)\right)$ be the associated connection as above. Let $F \subset S\left(k_{0}\right)$ be a copy of $\tau$ embedded in $S\left(k_{0}\right)$. There exists a 1 -dimensional space of column vectors $\left(f_{1}, \ldots, f_{d_{r}}\right)^{t}\left(f_{i} \in\right.$ $F)$ such that

$$
\left(f_{1}^{g}, \ldots, f_{d_{\tau}}^{g}\right)^{t}=M_{\tau}\left(g^{-1}\right)\left(f_{1}, \ldots, f_{d_{\tau}}\right)^{t}
$$


and $T_{\xi}\left(k_{0}\right) f=0 \forall i$ implies $\nabla_{\xi}^{\tau}\left(k_{0}\right)\left(f_{1}, \ldots, f_{d_{\tau}}\right)^{t}=0$. Hence $\sigma=\left(f_{1}, \ldots, f_{d_{\tau}}\right)^{t}$ is in $\left(\mathscr{V}^{\tau}\right) \nabla^{\tau}\left(k_{0}\right)$ and because of $(6.1)$ we see that $\sigma$ is fixed for the monodromy action. This sets up a one-to-one correspondence between $\left(Y_{\tau}\left(k_{0}\right)\right)^{\text {triv }}\left(=\left(\overline{X_{\tau}}\right)^{\text {triv }}\right)$ and vectors $\left(f_{1}, \ldots, f_{d_{\tau}}\right)^{t}$ of elements $f_{i} \in S\left(k_{0}\right)$ satisfying (6.1). Namely, suppose on the other hand that we are given $\sigma=\left(f_{1}, \ldots, f_{d_{\tau}}\right)^{t} \in\left(\mathscr{V}^{t}\right)^{\nabla^{\tau}\left(k_{0}\right)}$ that is trivial for monodromy. The exponents along the walls for the equation $\nabla_{\xi}^{\tau}\left(k_{0}\right) \sigma=0$ are 0 and $-2 k$. Since we assume that $k \notin \frac{1}{2} \mathbf{N}$ we may conclude that $f_{i}$ extends holomorphically along the walls, and that (6.1) holds. Finally we consider the equation $\left(\sum_{i=1}^{N} x_{i} \nabla_{i}^{\tau}\left(k_{0}\right)\right) \sigma=0$. It follows that $f_{i}$ is homogeneous of degree $-k_{0} \lambda_{\tau}$. Hence $-k_{0} \lambda_{\tau} \in \mathbf{N}, f_{i} \in \mathscr{P}_{-k_{0} \lambda_{r}}$, and $f_{i}$ satisfies $T_{\xi}\left(k_{0}\right) f_{i}=0$ $(\forall i)$. The result follows easily from this one-to-one correspondence.

Corollary 6.6. If $\tau \neq$ triv then $\operatorname{dim}_{\mathbf{C}}\left(S_{m, \tau}\right) \leq d_{\tau} \delta_{\tau}\left(\exp \left(\frac{2 \pi i m}{\lambda_{\tau}}\right)\right)$. Hence $m \mapsto$ $\operatorname{dim}_{\mathbf{C}}\left(S_{m, \tau}\right)$ is bounded by a function that is periodic in $m$ with period $\lambda_{\tau}$.

Proof. $S_{m, \tau}=S_{\tau}\left(-\frac{m}{\lambda_{\tau}}\right)$.

Remark 6.7. We hope that in fact equality holds. This would be very satisfying, since this would mean that the equations of Corollary 3.8 completely describe the decomposition of $(U)_{\mathscr{K}}$.

\section{Examples 6.8.}

1. $\tau=$ sign representation. Then $\delta_{\tau}(q)=1$ if $q=-1$, and zero otherwise. Moreover $\lambda_{\tau}=|R|$, so $q=\exp \left(\frac{2 \pi i m}{\lambda_{\tau}}\right)=-1$ iff $m=\left(p-\frac{1}{2}\right)|R| \quad(p \in \mathbf{N})$. On the other hand, if we let $\pi=\prod_{\alpha \in R_{+}} \alpha^{*}$ denote the fundamental alternating polynomial, then

$$
T_{\xi}\left(\frac{1}{2}-p\right) \pi^{2 p-1}=\left(\partial_{\xi}-(1-2 p) \sum_{\alpha \in R_{+}}(\alpha, \xi) \frac{1}{\alpha^{*}}\right) \pi^{2 p-1}=\left(\pi^{2 p-1} \partial_{\xi}\right) 1=0 .
$$

Hence $\operatorname{dim}_{\mathrm{C}}\left(S_{m, \tau}\right)=1$ if $m=\left(p-\frac{1}{2}\right)|R|$ and zero otherwise.

2. The dihedral cases. According to [CIK], the two-dimensional representations for $H_{I_{2}(m)}(q)$ can be defined explicitly as follows. Let $\sigma_{1}, \sigma_{2}$ be the standard generators. Then put $\pi_{j}\left(\sigma_{1}\right)=\left(\begin{array}{ll}1 & -q \\ 0 & -q\end{array}\right)$ and $\pi_{j}\left(\sigma_{2}\right)=\left(\begin{array}{cc}-q & 0 \\ -2-\left(\zeta^{j}+\zeta^{-j}\right) & 1\end{array}\right)$ where $\zeta=\exp \left(\frac{2 \pi i}{m}\right)$ and $j=1, \ldots, \frac{m-1}{2}$ (if $m$ is odd) or $j=1, \ldots, \frac{m}{2}-1$ (if $m$ is even). From this we find that $\delta_{\pi_{j}(q)=1}$ if $q=\zeta^{ \pm j}$ and zero otherwise (if $q=\zeta^{ \pm j}$ then $\left.\pi_{j}\left(\sigma_{i}\right)(q, q+1)^{t}=-q(q, q+1)^{t}(i=1,2)\right)$. Furthermore, it is easy to see that $\lambda_{\pi_{j}}=m(\forall j)$. Hence we infer from $q_{j}^{ \pm}=\zeta^{ \pm j}=\exp \left(-2 \pi i\left(\frac{m_{j}^{ \pm}}{m}\right)\right)$ that $m_{j}^{ \pm}=m p \pm j \quad(p \in \mathbf{N})$. By Corollary 6.6 we see that $\pi_{j}$ can occur in $S_{n}$ only if $n=m p \pm j$ for some $p \in \mathbf{N}$ and that the multiplicity is at most 1 . According to the results of $\S 5$ this multiplicity equals 1 for all $n$ of the form $n=m p \pm j$ $(p \in \mathbf{N})$. Hence. equality holds in Corollary 6.6 in this example.

3. The symmetric groups. We discuss some conjectures for the irreducible representations of $G=S_{n}$ (the Weyl group of type $A_{n-1}$ ) which appear in the space of singular polynomials. Recall that such representations correspond to partitions $\mu=\left(\mu_{1}, \mu_{2}, \ldots, \mu_{j}\right)$ with $\mu_{i} \in \mathbf{Z}_{+}, \mu_{1} \geq \mu_{2} \geq \cdots \geq \mu_{j}>0$ and $n=|\mu|=\sum_{i} \mu_{i}$. We will use $\mu$ as the label for the representation so that $\chi_{(n)}=1$ and $\chi_{(1,1, \ldots, 1)}$ is the sign character. 
The conjecture is that the partitions $\mu$ for which $S_{\mu}\left(k_{0}\right) \neq(0)$ for some $k_{0}$ are those with three or more (nonzero) parts of the form $\mu=(s d-1, d-$ $1, \ldots, d-1, r)$ with $s=1,2,3, \ldots, 0<r \leq d-1$ and $k_{0}=-j / d$ with $\operatorname{gcd}(j, d)=1 \quad(j \geq 1)$, and all of the two-part partitions $\left(\mu_{1}, \mu_{2}\right)$ with $k_{0}=$ $-j /\left(\mu_{1}+1\right)$ for $\operatorname{gcd}\left(j, \mu_{1}+1\right)<\left(\mu_{1}+1\right) / \mu_{2}$. Here gcd denotes greatest common divisor.

The evidence for this conjecture comes from the Specht modules for the Hecke algebra $H_{S_{n}}(q)$ constructed by Dipper and James [DJ1, DJ2]. Because of our different defining relations (we use generators $T_{1}, T_{2}, \ldots, T_{n-1}$ satisfying $T_{i}^{2}=q 1+(1-q) T_{i}, T_{i} T_{i+1} T_{i}=T_{i+1} T_{i} T_{i+1} \quad(1 \leq i \leq n-2), T_{i} T_{j}=T_{j} T_{i}$ for $1 \leq i<i+2 \leq j \leq n-1$, so that the eigenvalues of the generators are 1 and $-q$, rather than $q$ and -1 ), our partition labels are the transposes of those in [DJ1, DJ2].

For each partition $\mu$, Dipper and James construct an $H_{S_{n}}(q)$-module $M^{\mu}$ whose basis vectors correspond to certain tableaux with shape $\mu$, and then an irreducible submodule $S^{\mu}$, the Specht module. The action of the generators $T_{i}$ on this module is defined by the so-called Garnir relations. We are concerned with the existence of an $H_{S_{n}}(q)$-invariant element of the dual of $S^{\mu}$, that is, one which annihilates $v-T_{i} v$ for each basis element $v$ of $S^{\mu}$ and $1 \leq i \leq n-1$. From the Garnir relations one can show that the desired property holds exactly when the Gaussian polynomial (in the parameter $q$ ) $\left[\begin{array}{c}\mu_{j}+1 \\ i\end{array}\right]_{q}=0$ for each (nonzero) part $\mu_{j}$ and $1 \leq i \leq \mu_{j+1}$ (provided $\left.\mu_{j+1}>0\right)$. The coefficient of $q^{l}$ in the Gaussian polynomial $\left[\begin{array}{c}m \\ i\end{array}\right]_{q}=\prod_{l=1}^{i}\left(\frac{q^{m+1-l}-1}{q^{l}-1}\right)$ is the number of subsets $E$ of size $i$ of the set $\{1,2, \ldots, m\}$ for which $\mid\left\{\left(n_{1}, n_{2}\right) \mid n_{1} \in E, n_{2} \in E^{c}, n_{1}>\right.$ $\left.n_{2}\right\} \mid=l$. This is related to the action of $T_{i}$ on $S^{\mu}$.

For a partition $\mu$, let $Z(\mu)$ be the common zero set of $\left\{\left[\begin{array}{c}\mu_{j}+1 \\ i\end{array}\right]_{q} \mid 1 \leq i \leq\right.$ $\left.\mu_{j+1}\right\}$. Then $Z(\mu)=\varnothing$ except for the partitions described above. Let $P(j)=$ $\left\{q \in \mathbf{C} \mid q^{j}=1\right.$ and $q^{s} \neq 1$ for any $\left.s=1,2, \ldots, j-1\right\}$ (that is, the primitive $j$ th roots of unity). For $\mu=(s d-1, d-1, \ldots, d-1, r)$ (with $s \geq 1,0<$ $r \leq d-1), Z(\mu)=P(d)$. For $\mu=\left(\mu_{1}, \mu_{2}\right), Z(\mu)$ is the union of all $P(d)$ such that $d$ divides $\left(\mu_{1}+1\right)$ and $d>\mu_{2}>0$.

It is not difficult to see that for each $n$ and $\mu_{1} \leq n-1$ and each divisor $d$ of $\mu_{1}+1$ there is exactly one partition $\mu(|\mu|=n)$ with $P(d) \subset Z(\mu)$, and so each zero of $\left(q^{\mu_{1}+1}-1\right) /(q-1)$ is associated to a unique $\mu$. Recall the equation $q=-e^{2 \pi i k}$.

Several tasks remain to be done to prove our conjecture. One must establish a correspondence between the Specht modules and the monodromy representation $Y_{\mu}$ (see the beginning of $\S 6$ ), and further show that there is a function with trivial monodromy (a singular polynomial) exactly when the dual of the Specht module has an invariant vector, as decribed above.

Another problem is to prove the existence of singular polynomials in $S_{\mu}(k)$ for the appropriate degrees of homogeneity, that is, $-k \lambda_{\mu}$ (see (1.2) for the definition of $\left.\lambda_{\mu}\right)$. The zero-set of each factor of the Poincare series $\prod_{d=2}^{n}\left(\left(q^{d}-1\right) /(q-1)\right)$ of $S_{n}$ corresponds to the union of zero-sets $Z(\mu)$ with $\mu_{1}=d-1$. By use of Young's formula we see that

$$
\lambda_{\mu}=\left(n-\mu_{1}\right)\left(\left(\mu_{1}+1\right)+l\left(\mu_{2}+1\right)\right)-\mu_{2}\left(\mu_{2}+1\right) l(l+1) / 2
$$

for $\mu=\left(\mu_{1}, \mu_{2}, \ldots, \mu_{2}\right)$ with $(l+1)$ parts or $\mu=\left(\mu_{1}, \mu_{2}, \ldots, \mu_{2}, r\right)$ with 
$(l+2)$ parts, and this supports the conjectured link to the singular values $-j / d$, $\operatorname{gcd}(j, d)=1\left(\right.$ when $\left.\mu_{1}=s d-1, \mu_{2}=d-1\right)$.

\section{REFERENCES}

[B] E. Brieskorn, Die Fundamentalgruppe des Raumes der regulären Orbits einer komplexen Spiegelungs gruppe, Invent. Math. 12 (1971), 57-61.

[CIK] C. W. Curtis, N. Iwahori, and R. W. Kilmoyer, Hecke algebras and the characters of parabolic type of finite groups with BN-pairs, Publ. Math. Inst. Hautes Etudes Sci. 40 (1971), 81-116.

[D1] C. F. Dunkl, Reflection groups and orthogonal polynomials on the sphere, Math. Z. 197 (1988), 33-60.

[D2] - Differential-difference operators associated to reflection groups, Trans. Amer. Math. Soc. 311 (1989), 167-183.

[D3] - Operators commuting with Coxeter group actions on polynomials, Invariant Theory and Tableaux (D. Stanton, ed.), Springer-Verlag, 1990, pp. 107-117.

[D4] _ Integral kernels with reflection group invariance, Canad. J. Math. 43 (1991), 12131227.

[D5] - Differential-difference operators and monodromy representations of Hecke algebras, Pacific J. Math. 159 (1993), 271-298.

[DJ1] R. Dipper and G. James, Representations of Hecke algebras of general linear groups, Proc. London Math. Soc. (3) 52 (1986), 20-52.

[DJ2] - Blocks and idempotents of Hecke algebras of general linear groups, Proc. London Math. Soc. (3) 54 (1987), 57-82.

[Fe] W. Feit, The representation theory of finite groups, North-Holland, 1982.

[Fu] W. Fulton, Algebraic curves, Addison-Wesley, 1989.

[GU] A. Gyoja and K. Uno, On the semisimplicity of Hecke algebras, J. Math. Soc. Japan 41 (1989), 75-79.

[H] G. J. Heckman, A remark on the Dunkl differential-difference operators, Harmonic Analysis on Reductive Groups (W. Barker and P. Sally, eds.), Birkhaüser, Basel, 1991, pp. 181-191.

[M] I. G. Macdonald, The Poincaré series of a Coxeter group, Math. Ann. 199 (1972), 161-174.

[O] E. M. Opdam, Dunkl operators, Bessel functions and the discriminant of a finite Coxeter group, Comput. Math. 85 (1993), 333-373.

[S] T. A. Springer, Regular elements of finite reflection groups, Invent. Math. 25 (1974), 159198.

[Y] H. Yamane, Irreducible projective modules of the Hecke algebras of finite Coxeter groups, J. Algebra 127 (1989), 373-384.

(C. F. Dunkl) Department of Mathematics, University of Virginia, Charlottesville, VIRGINIA 22903

E-mail address: cfd5z@virginia.edu

(M. F. E. de Jeu and E. M. Opdam) Department of Mathematics, University of Leiden, P. O. Box 9512, 2300 RA LeIDEN, The Netherlands

E-mail address: jeu@rulwinw.leidenuniv.nl

E-mail address: opdam@rulwinw.leidenuniv.nl 